\title{
Publikasieaankondigings / Publication Announcements
}

English Dictionary for South Africa. 2011, 1108 pp. ISBN 978-1-86890-119-7 (Hardback with CD-ROM). Cape Town: Pharos Dictionaries. Price: R375.

Arnold Fischer, et al. Oxford English-Xhosa Dictionary. 2012, 752 pp. ISBN 9780 199043422 (Paperback). Goodwood: Oxford University Press Southern Africa. Price: R199.95.

Pedro A. Fuertes-Olivera and Henning Bergenholtz (Editors). e-Lexicography. The Internet, Digital Initiatives and Lexicography. 2011, xiv + 341 pp. ISBN 978-1-4411-2806-5 (Hardback). London/New York: Continuum International Publishing. Price: $£ 85.00$.

Lorna Hiles (Editor). Oxford South African Illustrated Dictionary. 2011, 384 pp. ISBN 978019599579 (Hardback). Goodwood: Oxford University Press Southern Africa. Price: R104.95.

Phillip Louw (Hoofredakteur). Oxford Afrikaanse Skoolwoordeboek. 2012, 684 pp. ISBN 9780195997958 (Paperback). Goodwood: Oxford University Press Southern Africa. Price: R109.95.

Jana Luther (Redakteur). 2011. Longman-HAT Basic Dictionary/Basiswoordeboek English-Afrikaans/Afrikaans-Engels. 2011, xiv + 224 pp. ISBN 978-1-77578138-7 (Sagteband). Pinelands: Pearson Education SA. Prys: R50.

Jana Luther (Redakteur). 2011. Longman-HAT Pocket Dictionary/Sakwoordeboek English-Afrikaans/Afrikaans-Engels. 2011, xiv + 558 pp. ISBN 978-1-77578126-4 (Sagteband). Pinelands: Pearson Education SA. Prys: R90.

Jana Luther (Redakteur). 2011. Longman-HAT School Dictionary/Skoolwoordeboek English-Afrikaans/Afrikaans-Engels. 2011, xiv + 558 pp. ISBN 978-1-77025780-1 (Sagteband). Pinelands: Pearson Education SA. Prys: R120.

Willy Martin (Hoofredakteur). Groot Woordeboek Afrikaans en Nederlands. 2011, 2228 pp. ISBN 978-1-86890-120-3 (Hardeband). Kaapstad: Pharos Woordeboeke, Prys: R999.95. (Artikel in hierdie uitgawe.).

Luvuyo S. Martins and Pumlani Sibula. Cricket Terminology/iSigama seQakamba/ Krieketterminologie. 2011, iv + 71 pp. ISBN 978-0-620-500289 (Paperback). Stellenbosch: Stellenbosch University Language Centre/IZiko leeLwimi le Univesithi yaseStellenbosch/Universiteit Stellenbosch Taalsentrum. 
Luvuyo Martins and Mkhonto van Zyl. Masincokole ngeMpilo ngesiXhosa./Let's chat about health in isiXhosa./Kom ons gesels oor gesondheid in isiXhosa. 2011, ix + 137 pp. ISBN 978-0-620-51030-1 (Paperback). Stellenbosch: IZiko leeLwimi le Univesithi yaseStellenbosch/Stellenbosch University Language Centre/Universiteit Stellenbosch Taalsentrum.

Grant Ngidi and Pumlani Sibula. Rugby Terminology/iSigama soMbhoxo/Rugbyterminologie. 2011, iv + 84 pp. ISBN 978-0-620-50029-6 (Paperback). Stellenbosch: Stellenbosch University Language Centre/IZiko leeLwimi le Univesithi yaseStellenbosch/Universiteit Stellenbosch Taalsentrum.

Helga Schaberg and Phillip Louw. Oxford South African School Dictionary. Third edition. CD-ROM. 2011. ISBN 9780199040643 (Paperback with CDROM). Goodwood: Oxford University Press Southern Africa. Price: R189.95.

South African Trilingual Wine Industry Dictionary/Suid-Afrikaanse Drietalige Wynbedryfwoordeboek/Isichazi-magama saseMzantsi Afrika seshishini lewayini ngeelwimi ezintathu. http://www.sawis.co.za/dictionary

Dennis Spohr. Towards a Multifunctional Lexical Resource. Design and Implementation of a Graph-based Lexicon Model. 2012, XIV + 206 pp. ISBN 978-3-11027115-7 (Hardback). Lexicographica. Series Maior 141. Berlin/New York: Walter de Gruyter. Price: $€ 89.95$.

Martin Stark. Bilingual Thematic Dictionaries. 2011, IX + 486 pp. ISBN 978-3-11025889-9 (Hardback). Lexicographica. Series Maior 140. Berlin/New York: Walter de Gruyter. Price: €99.95.

Tweetalige Skoolwoordeboek/Bilingual School Dictionary. 2012, 672 pp. ISBN 978-186890-128-9 (Sagteband). Kaapstad: Pharos Woordeboeke. Prys: R129.95. 